\title{
NOTE ON POISSON COHOMOLOGY ON WEIL BUNDLES
}

\author{
BASILE GUY RICHARD BOSSOTO, OLIVIER MABIALA MIKANOU, \\ AND APÉPÉ JUGENDÈNE NGUÉNGUÉ LOUVOUANDOU
}

\begin{abstract}
The purpose of this note on Poisson cohomology is to show that, if $M$ is a Poisson manifold and if $A$ is a Weil algebra, then the Weil bundle $M^{A}$ is a Poisson manifold. Therefore, we establish a relationship between Poisson cohomology $H_{\{,,\}_{A}}^{\bullet}\left(M^{A}, A\right)$ with values in $A$ and Poisson cohomology $H_{\{,,,\}, \mathbb{R}}^{\bullet}\left(M^{A}\right)$ with real values.
\end{abstract}

\section{INTRODUCTION}

In this work, $M$ denotes a smooth manifold of dimension $n, C^{\infty}(M)$ the algebra of smooth functions on $M$ and $A$ a Weil algebra, i.e a real commutative, associative, unitary algebra of finite dimension, of the form $A=\mathbb{R} \oplus \mathfrak{m}$, where $\mathfrak{m}$ is a nilpotent ideal.

We recall that an infinitely near point to $x \in M$ of type $A$ is a homomorphism of $\mathbb{R}$-algebras

$$
\xi: C^{\infty}(M) \longrightarrow A
$$

such that $\xi(f)-f(x) \in \mathfrak{m}$, for all $f \in C^{\infty}(M)$ [13]. We denote by $M_{x}^{A}$ the set of all infinitely near points to $x \in M$ of type $A$ and by

$$
M^{A}=\bigcup_{x \in M} M_{x}^{A}
$$

the manifold of near points of $M$ of type $A$ or simply the Weil bundle of $M$ of type $A$.

We denote by $\pi_{M}: M^{A} \longrightarrow M$, the projection which assigns every infinitely near point to $x \in M$ to its origin $x$. The triple $\left(M^{A}, \pi_{M}, M\right)$ defines the bundle of infinitely near points or simply Weil bundle [13], [8], [6].

We denote by $C^{\infty}\left(M^{A}\right)$ the algebra of smooth functions on $M^{A}$ (with values in $\mathbb{R}$ ) and $C^{\infty}\left(M^{A}, A\right)$ the algebra of smooth functions on $M^{A}$ with values in $A$. The $C^{\infty}(M)$-module of vector fields on $M$ is denoted by $\mathfrak{X}(M)$. If $M$ is provided with a Poisson structure with bracket $\{\cdot, \cdot\}$, we establish an isomorphism between the representations from $C^{\infty}(M)$ into $C^{\infty}\left(M^{A}, A\right)$, and from $C^{\infty}\left(M^{A}, A\right)$ into $C^{\infty}\left(M^{A}, A\right)$, respectively, defined in [9].

When both $M$ and $N$ are smooth manifolds and when $h: M \longrightarrow N$ is a smooth mapping, then the mapping

$$
h^{A}: M^{A} \longrightarrow N^{A}, \xi \longmapsto h^{A}(\xi),
$$

2010 Mathematics Subject Classification. Primary 53D17 ; Secondary 58A20.

Key words and phrases. Weil algebra, Weil bundle, Poisson manifolds, Cohomology. 
such that, for any $g \in C^{\infty}(N)$,

$$
\left[h^{A}(\xi)\right](g)=\xi(g \circ h)
$$

is also smooth. When $h$ is a diffeomorphism, it is the same for $h^{A}$.

The set $C^{\infty}\left(M^{A}, A\right)$ of smooth functions on $M^{A}$ with values in $A$ is a commutative, unitary algebra over $A$. By identifying $\mathbb{R}^{A}$ with $A$, for $f \in$ $C^{\infty}(M)$, the mapping

$$
f^{A}: M^{A} \longrightarrow A, \xi \longmapsto \xi(f)
$$

is differentiable and the mapping

$$
C^{\infty}(M) \longrightarrow C^{\infty}\left(M^{A}, A\right), f \longmapsto f^{A},
$$

is an injective homomorphism of algebras and we have:

$$
(f+g)^{A}=f^{A}+g^{A} ;(\lambda \cdot f)^{A}=\lambda \cdot f^{A} ;(f \cdot g)^{A}=f^{A} \cdot g^{A}
$$

for $\lambda \in \mathbb{R}, f, g \in C^{\infty}(M)$.

We denote by $\mathfrak{X}\left(M^{A}\right)$, the set of all vector fields on $M^{A}$. According to [2], we have the following equivalent assertions:

(1) A vector field on $M^{A}$ is a differentiable section of the tangent bundle $T M^{A}$.

(2) A vector field on $M^{A}$ is a derivation of $C^{\infty}\left(M^{A}\right)$.

(3) A vector field on $M^{A}$ is a linear mapping $X: C^{\infty}(M) \longrightarrow C^{\infty}\left(M^{A}, A\right)$ such that

$$
X(f \cdot g)=X(f) \cdot g^{A}+f^{A} \cdot X(g), \quad \text { for any } f, g \in C^{\infty}(M) .
$$

Thus, the set $\mathfrak{X}\left(M^{A}\right)$ of vector fields on $M^{A}$ considered as derivations of $C^{\infty}(M)$ into $C^{\infty}\left(M^{A}, A\right)$ is a module over $C^{\infty}\left(M^{A}, A\right)$.

When

$$
\theta: C^{\infty}(M) \longrightarrow C^{\infty}(M)
$$

is a vector field on $M$, then the mapping

$$
\theta^{A}: C^{\infty}(M) \longrightarrow C^{\infty}\left(M^{A}, A\right), f \longmapsto[\theta(f)]^{A}
$$

is a vector field on $M^{A}$. We say that the vector field $\theta^{A}$ is the prolongation to $M^{A}$ of the vector field $\theta$.

If $X$ is a vector field on $M^{A}$, considered as a derivation of $C^{\infty}(M)$ into $C^{\infty}\left(M^{A}, A\right)$, then there exists, [2], a unique derivation

$$
\widetilde{X}: C^{\infty}\left(M^{A}, A\right) \longrightarrow C^{\infty}\left(M^{A}, A\right)
$$

such that:

$1 / \widetilde{X}$ is $A$-linear;

2/ $\widetilde{X}\left[C^{\infty}\left(M^{A}\right)\right] \subset C^{\infty}\left(M^{A}\right)$;

$3 / \tilde{X}\left(f^{A}\right)=X(f)$ for any $f \in C^{\infty}(M)$.

The mapping

$$
[\cdot, \cdot]: \mathfrak{X}\left(M^{A}\right) \times \mathfrak{X}\left(M^{A}\right) \longrightarrow \mathfrak{X}\left(M^{A}\right),(X, Y) \longmapsto \widetilde{X} \circ Y-\widetilde{Y} \circ X,
$$

is $A$-bilinear and defines a structure of $A$-Lie algebra on $\mathfrak{X}\left(M^{A}\right)$.

Let $\left(a_{\alpha}\right)_{\alpha=1, \ldots, r}$ be a basis of $A$ and $\left(a_{\alpha}^{*}\right)_{\alpha=1, \ldots, r}$ be the dual basis. 
If

$$
Y: C^{\infty}\left(M^{A}, A\right) \longrightarrow C^{\infty}\left(M^{A}, A\right)
$$

is an $A$-linear derivation such that

$$
Y\left(f^{A}\right)=\tilde{X}\left(f^{A}\right)
$$

for any $f \in C^{\infty}(M)$, then

$$
Y\left[C^{\infty}\left(M^{A}\right)\right] \subset C^{\infty}\left(M^{A}\right)
$$

since

$$
Y\left(a_{\alpha}^{*} \circ f^{A}\right)=\tilde{X}\left(a_{\alpha}^{*} \circ f^{A}\right) \in C^{\infty}\left(M^{A}\right)
$$

for any $\alpha=1,2, . ., r$. Thus, $Y=\widetilde{X}$.

1.1. Poisson structure on Weil bundle. A Poisson structure on a smooth manifold $M$ is defined as a bilinear bracket operation $\{\cdot, \cdot\}$ on $C^{\infty}(M)$ such that the pair $\left(C^{\infty}(M),\{\cdot, \cdot\}\right)$ is a real Lie algebra and

$$
\{f, g \cdot h\}=\{f, g\} \cdot h+g \cdot\{f, h\}
$$

for any $f, g, h \in C^{\infty}(M)$. We say in this case that $C^{\infty}(M)$ is a Poisson algebra and $M$ is a Poisson manifold [12, [7], [5].

For any $f \in C^{\infty}(M)$, the mapping

$$
\operatorname{ad}(f): C^{\infty}(M) \longrightarrow C^{\infty}(M), g \longmapsto\{f, g\},
$$

is a vector field on $M$ and, for any $g \in C^{\infty}(M)$, we have

$$
\operatorname{ad}(f g)=f \cdot \operatorname{ad}(g)+g \cdot \operatorname{ad}(f) .
$$

For any $f \in C^{\infty}(M)$, let

$$
[\operatorname{ad}(f)]^{A}: C^{\infty}(M) \longrightarrow C^{\infty}\left(M^{A}, A\right), g \longmapsto\{f, g\}^{A}
$$

be the prolongation of the vector field $\operatorname{ad}(f)$ and let

$$
\widetilde{\operatorname{ad}(f)}^{A}: C^{\infty}\left(M^{A}, A\right) \longrightarrow C^{\infty}\left(M^{A}, A\right)
$$

be the unique $A$-linear derivation such that

$$
\begin{aligned}
\widetilde{\operatorname{ad}(f)]^{A}}\left(g^{A}\right) & =[\operatorname{ad}(f)]^{A}(g) \\
& =\{f, g\}^{A}
\end{aligned}
$$

for any $g \in C^{\infty}(M)$.

For any $\varphi \in C^{\infty}\left(M^{A}, A\right)$, the mapping

$$
\tau_{\varphi}: C^{\infty}(M) \longrightarrow C^{\infty}\left(M^{A}, A\right), f \longmapsto-[\widetilde{\operatorname{ad}(f)}]^{A}(\varphi),
$$

is a vector field on $M^{A}$. We denote by

$$
\widetilde{\tau_{\varphi}}: C^{\infty}\left(M^{A}, A\right) \longrightarrow C^{\infty}\left(M^{A}, A\right)
$$

the unique $A$-linear derivation such that

$$
\widetilde{\tau_{\varphi}}\left(f^{A}\right)=\tau_{\varphi}(f)
$$

for any $f \in C^{\infty}(M)$. We have

$$
\widetilde{\tau}_{f^{A}}=\widetilde{\operatorname{ad}(f)]^{A}}
$$


and for all $\varphi, \psi \in C^{\infty}\left(M^{A}, A\right)$ and for $a \in A$ :

$$
\widetilde{\tau_{\varphi+\psi}}=\widetilde{\tau_{\varphi}}+\widetilde{\tau_{\psi}} ; \widetilde{\tau_{a \cdot \varphi}}=a \cdot \widetilde{\tau_{\varphi}} ; \widetilde{\tau_{\varphi \cdot \psi}}=\varphi \cdot \widetilde{\tau_{\psi}}+\psi \cdot \widetilde{\tau_{\varphi}}
$$

For any $\varphi, \psi \in C^{\infty}\left(M^{A}, A\right)$, if we let

$$
\{\varphi, \psi\}_{A}=\widetilde{\tau_{\varphi}}(\psi),
$$

this bracket $\{\cdot, \cdot\}_{A}$ defines a structure of Poisson $A$-algebra on $C^{\infty}\left(M^{A}, A\right)$ i.e the mapping

$$
\{\cdot, \cdot\}_{A}: C^{\infty}\left(M^{A}, A\right) \times C^{\infty}\left(M^{A}, A\right) \longrightarrow C^{\infty}\left(M^{A}, A\right),(\varphi, \psi) \longmapsto\{\varphi, \psi\}_{A},
$$

is $A$-bilinear and skew symmetric and for any $\varphi, \psi, \phi \in C^{\infty}\left(M^{A}, A\right)$,

$$
\{\varphi, \psi \cdot \phi\}_{A}=\{\varphi, \psi\}_{A} \cdot \phi+\psi \cdot\{\varphi, \phi\}_{A}
$$

$$
\left\{\varphi,\{\psi, \phi\}_{A}\right\}_{A}+\left\{\psi,\{\phi, \varphi\}_{A}\right\}_{A}+\left\{\phi,\{\varphi, \psi\}_{A}\right\}_{A}=0
$$

We say that the structure of $A$-Poisson manifold on $M^{A}$ defined by $\{\cdot, \cdot\}_{A}$ is the prolongation on $M^{A}$ of the structure of Poisson manifold on $M$ defined by $\{\cdot, \cdot\}[1]$.

\section{Poisson cohomology on Weil Bundle}

In [10], E. Okassa showed that when $\varphi \in A^{*}$ is a $\mathbb{R}$-linear form on $A$ and when the manifold $M$ is equipped with a symplectic structure $(M, \omega)$ ( a pseudo-Riemannian structure $(M, g)$, respectively), the lift $\varphi \circ \omega^{A}$ (respectively $\varphi \circ g^{A}$ ) on $M^{A}$ is a symplectic structure (a pseudo-Riemannian structure, repectively) if and only if the dimension of the annihilator of the maximal ideal $\mathfrak{m}, \operatorname{ann}(\mathfrak{m})$, is equal to 1 and $\varphi(\operatorname{ann}(\mathfrak{m})) \neq 0$. In [11], V. V. Shurygin studied the Lifts of Poisson structures to $M^{A}$ when $A$ is a Frobenius algebra. In this paper, we show that if $M$ is a Poisson manifold, then $M^{A}$ is a Poisson manifold.

Let $(M,\{.,\}$.$) be a Poisson manifold,$

$$
\text { ad }: f \in C^{\infty}(M) \longmapsto\{f, \cdot\} \in \operatorname{Der}_{\mathbb{R}}\left(C^{\infty}(M)\right)
$$

the adjoint representation of this structure and $d_{\{. .,\}}$

$$
\cdots \longrightarrow \bigwedge_{\{., .\}, \mathbb{R}}^{\bullet} \stackrel{d_{\{., .\}}}{\bullet} \bigwedge_{\{., .\}, \mathbb{R}}^{\bullet+1} \longrightarrow \cdots
$$

the Lichnerowicz cohomology operator associated to the representation ad from the Lie-Poisson algebra $\left(C^{\infty}(M),\{.,\}.\right)$ into $\left(\operatorname{Der}_{\mathbb{R}}\left(C^{\infty}(M)\right),[.,].\right)[12$, where $\bigwedge_{\{.,,\}, \mathbb{R}}^{\bullet}=\bigwedge_{\{, .\}, \mathbb{R}}^{p}\left(C^{\infty}(M), C^{\infty}(M)\right)$ denotes the set of $p$-multilinear skew symmetric mappings from $\underbrace{C^{\infty}(M) \times \ldots \times C^{\infty}(M)}_{p \text {-times }}$ into $C^{\infty}(M)$.

It is well known that if the algebra $C^{\infty}(M)$ is a Poisson algebra with bracket $\{.,$.$\} (which is \mathbb{R}$-bilinear), then the algebra $C^{\infty}\left(M^{A}, A\right)$ is a Poisson algebra with bracket $\{., .\}_{A}$ (which is $A$-bilinear) [1]. The main object of this work is to define a $\mathbb{R}$-bilinear Poisson bracket on $C^{\infty}\left(M^{A}\right)=C^{\infty}\left(M^{A}, \mathbb{R}\right)$ 
i.e we show that if $(M,\{.,\}$.$) is a Poisson manifold then M^{A}$ is a Poisson manifold.

If $\operatorname{Der}_{A}\left(C^{\infty}\left(M^{A}, A\right)\right)$ denotes the set of derivations of $C^{\infty}\left(M^{A}, A\right)$ which are $A$-linear, the mapping

$$
\tau: C^{\infty}(M) \longrightarrow \operatorname{Der}_{A}\left(C^{\infty}\left(M^{A}, A\right)\right), f \longmapsto \tau(f)
$$

such that

$$
\tau(f)(\varphi)=\tau_{\varphi}(f) \text { for all } \varphi \in C^{\infty}\left(M^{A}, A\right)
$$

is a $\mathbb{R}$-representation from $\left(C^{\infty}(M),\{.,\}.\right)$ into $\left(C^{\infty}\left(M^{A}, A\right),\{., .\}_{A}\right)$.

This definition induces (step by step) the construction of the cohomology operator $\boldsymbol{d}$ associated with this representation, defined as follows: for all $p=0,1, \ldots$, for any element $\omega^{*} \in \bigwedge_{\{,,\}_{A}, \mathbb{R}}^{p}\left(C^{\infty}(M), C^{\infty}\left(M^{A}, A\right)\right)$, for all functions $f_{1}, \ldots, f_{p}, f_{p+1} \in C^{\infty}(M)$, we have

$$
\begin{gathered}
\boldsymbol{d} \omega^{*}\left(f_{1}, \ldots, f_{p+1}\right)=\sum_{j=1}^{p+1}(-1)^{j-1} \tau\left(f_{j}\right)\left[\omega^{*}\left(f_{1}, \ldots, \widehat{f}_{j}, \ldots, f_{p+1}\right)\right] \\
+\sum_{1 \leq k<\ell \leq p+1}(-1)^{k+\ell} \omega^{*}\left(\left\{f_{k}, f_{\ell}\right\}, f_{1}, \ldots, \widehat{f}_{k}, \ldots, \widehat{f}_{\ell}, \ldots, f_{p+1}\right)
\end{gathered}
$$

(the notation $\widehat{\cdot}$ means the omission of the component thus marked). This defines an element of $\bigwedge_{\{\ldots,\}_{A}, \mathbb{R}}^{p+1}\left(C^{\infty}(M), C^{\infty}\left(M^{A}, A\right)\right)$.

We denote by

$$
H_{\{\ldots,,\}, \mathbb{R}}^{p}\left(M^{A}, A\right), \quad p=0, \ldots, n
$$

the associated $\mathbb{R}$-cohomology algebras. As $\operatorname{dim} M=n$, these $\mathbb{R}$-vector spaces are zero as soon as $p \geq n+1$.

We can also consider the mapping

$$
\tilde{\tau}: \varphi \in C^{\infty}\left(M^{A}, A\right) \longmapsto \widetilde{\tau_{\varphi}} \in \operatorname{Der}_{A}\left(C^{\infty}\left(M^{A}, A\right)\right)
$$

as an $A$-representation from the Lie algebra $\left(C^{\infty}\left(M^{A}, A\right),\{., .\}_{A}\right)$ into the Lie algebra $\left(C^{\infty}\left(M^{A}, A\right),\{., .\}_{A}\right)$ and in the same way as before, we associate a cohomology operator

$$
\boldsymbol{d}_{A}: \bigwedge_{\{., .\}_{A}, A}\left(C^{\infty}\left(M^{A}, A\right), C^{\infty}\left(M^{A}, A\right)\right) \longrightarrow \bigwedge_{\{., .\}_{A}, A}^{\bullet+1}\left(C^{\infty}\left(M^{A}, A\right), C^{\infty}\left(M^{A}, A\right)\right)
$$


We define for all $\boldsymbol{\omega}^{*} \in \bigwedge_{\{., .\}_{A}, A}^{p} \operatorname{Hom}_{A}\left(C^{\infty}(M, A), C^{\infty}(M, A)\right)$, for all functions $\varphi_{1}, \ldots, \varphi_{p+1} \in C^{\infty}\left(M^{A}, A\right)$ :

$$
\begin{aligned}
& \boldsymbol{d} \boldsymbol{\omega}^{*}\left(\varphi_{1}, \ldots, \varphi_{p+1}\right)=\sum_{j=1}^{p+1}(-1)^{j-1} \widetilde{\tau_{\varphi}}\left[\boldsymbol{\omega}^{*}\left(\varphi_{1}, \ldots, \widehat{\varphi_{j}}, \ldots, \varphi_{p+1}\right)\right] \\
& +\sum_{1 \leq k<\ell \leq p+1}(-1)^{k+\ell} \boldsymbol{\omega}^{*}\left(\left\{\varphi_{k}, \varphi_{\ell}\right\}_{A}, \varphi_{1}, \ldots, \widehat{\varphi_{k}}, \ldots, \widehat{\varphi_{\ell}}, \ldots, \varphi_{p+1}\right) \\
& =\sum_{j=1}^{p+1}(-1)^{j-1}\left\{\varphi_{j}, \boldsymbol{\omega}^{*}\left(\varphi_{1}, \ldots, \widehat{\varphi_{j}}, \ldots, \varphi_{p+1}\right)\right\}_{A} \\
& +\sum_{1 \leq k<\ell \leq p+1}(-1)^{k+\ell} \boldsymbol{\omega}^{*}\left(\left\{\varphi_{k}, \varphi_{\ell}\right\}_{A}, \varphi_{1}, \ldots, \widehat{\varphi_{k}}, \ldots, \widehat{\varphi}_{\ell}, \ldots, \varphi_{p+1}\right) .
\end{aligned}
$$

The bracket $\{., .\}_{A}$ above is of course understood as extended in the sense of the Schouten-Nijenhuis bracket. The associated cohomology $A$-algebras will be denoted this time

$$
H_{\{. .,\}_{A}, A}^{p}\left(M^{A}, A\right), \quad p=0, \ldots, n .
$$

(Indeed $M^{A}$ is considered here with its $A$-manifold structure, these cohomology algebras are null as soon as $p>n+1)$.

Theorem 1. For all $p \in N$, the cohomology vector space over $\mathbb{R}, H_{\{., .\}, \mathbb{R}}^{p}\left(M^{A}, A\right)$ also has a structure of $A$-module and coincides with the cohomolgy module over $A, H_{\{.,\}_{A}, A}^{p}\left(M^{A}, A\right)$.

Proof. Consider the representation $\tau$ from $\left(C^{\infty}(M),\{.,\}.\right)$ into $\left(C^{\infty}\left(M^{A}, A\right),\{., .\}_{A}\right)$ defined by (2.1) and

$$
\rho:\left(C^{\infty}\left(M^{A}, A\right),\{\cdot, \cdot\}_{A}\right) \rightarrow\left(\operatorname{Der}_{A}\left(C^{\infty}\left(M^{A}, A\right)\right),[., .]\right)
$$

a representation of Poisson algebra $\left(C^{\infty}\left(M^{A}, A\right),\{., .\}_{A}\right)$. If we assume that

$$
\rho\left(f^{A}\right)=\tau(f) \quad \forall f \in C^{\infty}(M),
$$

we observe then that the representations $\rho$ and $\tilde{\tau}$ (defined by (2.3) coincide for all $\varphi=f^{A}, f \in C^{\infty}(M)$, therefore are equal since the functions $f^{A}$ generate $C^{\infty}\left(M^{A}, A\right)$ as $A$-module and that $\rho$ and $\tilde{\tau}$ are homomorphisms of $A$-modules. There is therefore a canonical isomorphism linking the representations $\tau$ and $\tilde{\tau}$ and which made it possible to construct the two complexes whose cohomology we want to compare: to $\tau$, we associate $\tilde{\tau}$ as we saw in the previous section; given on the other hand a representation: $\rho: C^{\infty}\left(M^{A}, A\right) \rightarrow \operatorname{Der}_{A}\left(C^{\infty}\left(M^{A}, A\right)\right)$, we associate the representation

$$
\underline{\rho}: C^{\infty}(M) \rightarrow \operatorname{Der}_{A}\left(C^{\infty}\left(M^{A}, A\right)\right), f \longmapsto \rho\left(f^{A}\right),
$$

thus defining a representation of $\left(C^{\infty}(M),\{.,\}.\right)$ in $\left(C^{\infty}\left(M^{A}, A\right),[.,].\right)$. If 2 elements $\omega_{0}^{*}$ and $\omega_{1}^{*}$ of $\bigwedge_{\{\ldots,\}_{A}, A}^{p}\left(C^{\infty}\left(M^{A}, A\right), C^{\infty}\left(M^{A}, A\right)\right)$ are cohomologous relative to the operator $d_{A}$, we find by testing them on $\left(f_{1}^{A}, \ldots, f_{p}^{A}\right)$ that the 
2-elements

$$
\left[\left(f_{1}, \ldots, f_{p}\right) \longmapsto \boldsymbol{\omega}_{j}^{*}\left(f_{1}^{A}, \ldots, f_{p}^{A}\right)\right] \in \bigwedge_{\{.,\}_{A}, \mathbb{R}}^{p}\left(C^{\infty}(M), C^{\infty}\left(M^{A}, A\right)\right), j=0,1,
$$

are cohomologous with respect to the cohomology operator $d$.

Conversely, given two elements $\alpha_{0}^{*}, \alpha_{1}^{*} \in \bigwedge_{\{., .\}_{A}, \mathbb{R}}^{p}\left(C^{\infty}(M), C^{\infty}\left(M^{A}, A\right)\right)$ assumed to be cohomologous with respect to the operator $\boldsymbol{d}$. The elements $\omega_{0}^{*}$ and $\omega_{1}^{*}$ defined by extending by $A$-linearity

$$
\left(f_{1}^{A}, \ldots, f_{p}^{A}\right) \longmapsto \alpha_{j}^{*}\left(f_{1}, \ldots, f_{p}\right) \quad\left(f_{1}, \ldots, f_{p} \in C^{\infty}(M)\right), j=0,1,
$$

are elements of $\bigwedge_{\{.,\}_{A}, A}^{\bullet}\left(C^{\infty}\left(M^{A}, A\right), C^{\infty}\left(M^{A}, A\right)\right)$ which are cohomologous via this time the operator $\boldsymbol{d}_{A}$.

The cohomology defined so will be denoted by the following $H_{\{. .,\}_{A}}^{\bullet}\left(M^{A}, A\right)$.

Proposition 2. The restriction of the bracket $\{., .\}_{A}$ to $C^{\infty}\left(M^{A}\right) \times C^{\infty}\left(M^{A}\right)$, is a Poisson bracket.

Proof. For all $\varphi \in C^{\infty}\left(M^{A}, A\right)$, the vector field $\tau_{\varphi}$ is $A$-linear by construction. Thus $\widetilde{\tau_{\varphi}}\left[C^{\infty}\left(M^{A}\right)\right] \subset C^{\infty}\left(M^{A}\right)$. If $F$ and $G$ are in $C^{\infty}\left(M^{A}\right)$, then $\widetilde{\tau_{F}}(G)=\{F, G\}_{A} \in C^{\infty}\left(M^{A}\right)$. We define thus a Poisson bracket on $C^{\infty}\left(M^{A}\right)$ by

$$
C^{\infty}\left(M^{A}\right) \times C^{\infty}\left(M^{A}\right) \longrightarrow C^{\infty}\left(M^{A}\right),(F, G) \longmapsto\{F, G\}_{A}=\widetilde{\tau_{F}}(G) .
$$

We have the following result:

Theorem 3. Let $(M,\{.,\}$.$) be a Poisson structure on M$ and $A$ a Weil algebra. Then $M^{A}$ is a Poisson manifold. In addition, we have

$$
H_{\{,,\}_{A}}^{\bullet}\left(M^{A}, A\right)=A \otimes_{\mathbb{R}} H_{\{,,,\}, \mathbb{R}}^{\bullet}\left(M^{A}, \mathbb{R}\right) .
$$

Proof. According to the previous Proposition, the restriction of the bracket $\{., .\}_{A}$ to $C^{\infty}\left(M^{A}\right) \times C^{\infty}\left(M^{A}\right)$ is a Poisson bracket. Thus, $M^{A}$ is a Poisson manifold with bracket $\{., .\}_{A}$ restricted to $C^{\infty}\left(M^{A}\right) \times C^{\infty}\left(M^{A}\right)$.

When $\left\{e_{1}, \ldots, e_{k}\right\}$ denotes a basis of $A$ as $\mathbb{R}$-module of dimension $k$, we now introduce canonical $A$-isomorphism

$$
\sigma: \varphi \in C^{\infty}\left(M^{A}, A\right) \longmapsto \sigma(\varphi) \in A \otimes_{\mathbb{R}} C^{\infty}\left(M^{A}\right)
$$

associating with $\varphi: x \mapsto \sum_{j=1}^{k} \varphi_{j}(x) e_{j}$ the element $\varphi_{j} \otimes e_{j}$, where $\varphi=$ $\sum_{j=1}^{k} \varphi_{j} e_{j}$ and $\varphi_{j} \in C^{\infty}\left(M^{A}, \mathbb{R}\right)$.

Any $p$-form $\boldsymbol{\omega}^{*} \in \bigwedge_{\{. .,\}_{A}}^{p}\left(C^{\infty}\left(M^{A}, A\right), C^{\infty}\left(M^{A}, A\right)\right)$ of degree $p$ is equivalent via this isomorphism to the given of $\bigwedge^{p}\left(A \otimes_{\mathbb{R}} C^{\infty}\left(M^{A}\right), A \otimes_{\mathbb{R}} C^{\infty}\left(M^{A}\right)\right)$. 
By passing to the quotient relative to the cohomology induced by the operator $\boldsymbol{d}_{A}$, we deduce the isomorphisms between the desired cohomology groups. For this purpose, we associate with the class of $\boldsymbol{\eta}^{*}$ the class of

$$
\left(\tilde{f}_{1}, \ldots, \tilde{f}_{p}\right) \in\left(A \otimes C^{\infty}\left(M^{A}\right)\right)^{p} \longmapsto \sigma \circ \boldsymbol{\eta}^{*}\left(\sigma^{-1}\left(\tilde{f}_{1}\right), \ldots, \sigma^{-1}\left(\tilde{f}_{p}\right)\right)
$$

This completes the Proof.

Acknowledgements. The authors thank Professor Eugène Okassa and Professor Alain Yger for their comments, remarks and suggestions as well as International Mathematical Union (IMU) for the scholarship granted to Olivier Mabiala Mikanou.

\section{REFERENCES}

[1] B.G.R. Bossoto, E. Okassa, A-Poisson structures on Weil bundles, Int. J. Contemp. Math. Sciences, Vol. 7(2012), pp. 785-803.

[2] B.G.R. Bossoto, E. Okassa, Champs de vecteurs et formes différentielles sur une variété des points proches, Arch. Math (Brno), 44 (2008), pp. 159-171.

[3] N. Bourbaki, Algèbres, Chapitres 1 à 3, Hermann, Paris 1970.

[4] N. Bourbaki, Algèbres, Chapitres 10, Algèbre homologique, Masson, Paris, New York, Barcelone, Milan, 1980.

[5] P. Caressa, The algebra of Poisson brackets, Young Algebra Seminar, Roma Tor Vergata, 2000.

[6] I. Kolar, P.W. Michor and J. Slovak, Natural Operations in Differential Geometry, Springer-Verlag, Berlin, 1993.

[7] A. Lichnerowicz, Les variétés de Poisson et leurs algèbres de Lie associées, J. Diff Géom., 12 (1977)., pp. 253-300.

[8] A. Morimoto, Prolongation of connections to bundles of infinitely near points, J. Differential Geom. 11 (1976), pp. 479-498.

[9] B.V. Nkou, B.G.R, Bossoto, Cohomology associated to a Poisson structure on Weil bundles, Int. Math. Forum, vol. 9, no. 7 (2014), pp. 305-316.

[10] E. Okassa, Relèvements de structures symplectiques et pseudoriemanniennes à des variétés de points proches, Nagoya Math. J., 115, (1989), 63-71.

[11] V. V. Shurygin, jr, Lifts of Poisson structures to Weil bundles, Lobachevskii Journal of Mathematics, 2012, Volume 33, Issue 1, pp $75-107$.

[12] I. Vaisman, Lectures on the Geometry of Poisson manifolds, in Progress in Math., 118, (1994) Basel Birkhäuser.

[13] A. Weil, Théorie des points proches sur les variété différentiables, Colloque Géom. Diff. Strasbourg, 1953, pp. 111-117. 
Department of Mathematics, Faculty of Sciences and Technology, Marien Ngouabi University, and, Institut de Recherche en Sciences Exactes et Na-

turelles (IRSEN), Box: 69, Brazzaville, Congo

E-mail address: bossotob@yahoo.fr

Department of Mathematics, Faculty of Sciences and Technology, Marien Ngouabi University, Box: 69, Brazzaville, Congo

E-mail address: stive.elg@gmail.com

Department of Mathematics, Faculty of Sciences and Technology, Marien Ngouabi University, Box: 69, Brazzaville, Congo

E-mail address: nguengueapepe@gmail.com 